Saratov, Russian Federation; ${ }^{1}$ Saratov State Medical University name of V.I. Razumovsky Ministry of Health of Russia, Hospital Therapy, Saratov, Russian Federation

Background: Rheumatoid arthritis (RA) is a chronic autoimmune disease that leads to joint damage and deformation. Pain syndrome, along with functional limitations, causes the emergence of anxiety-depressive disorders. The patient's psycho-emotional characteristics affect the patient's quality of life and the effectiveness of the therapy. The aim: to assess the severity of anxiety and depression in women with rheumatoid arthritis, depending on the type of the pain syndrome.

Objectives: The study included 163 women with RA according to the EULAR / ACR 2010 criteria (age 53,9 $\pm 10,15$ years, RA duration - 10 [4; 14] years, DAS28 $-5,03[4,35 ; 5,8])$.

Methods: We used the Hospital Depression and Anxiety Scale (HADS) questionnaire: 0-7 points were assessed as the absence of significant symptoms of anxiety and depression, 8-10 points - subclinically expressed anxiety and depression, more than 11 points - clinically expressed anxiety and depression. The severity of pain was determined by the VAS: no pain $(0-4 \mathrm{~mm})$, mild pain $(5-44 \mathrm{~mm})$, moderate pain $(45-74 \mathrm{~mm})$, severe pain $(75-100 \mathrm{~mm})$. Assessment of the type of pain (identification of the neuropathic component of pain) was carried out using the DN4 questionnaire: a sum of 4 or more points indicated the presence of a neuropathic component of pain (NCP). Statistical processing was performed using the STATISTICA 10,0 program.

Results: The frequency of occurrence of anxiety-depressive disorders in RA patients was determined: clinically pronounced anxiety was detected in 35 $(21,4 \%)$ patients, depression - in $34(20,9 \%)$; subclinically expressed anxiety - in $42(25,8 \%)$, depression - in $44(27 \%)$ patients; absence of reliably pronounced symptoms of anxiety - in $86(52,8 \%)$ patients, depression - in 85 $(52,1 \%)$ patients.

Severe pain according to VAS was noted in 57 (35\%) patients, moderate pain - in 75 (46\%), in 31 (19\%) patients the pain syndrome was mild. In $81(49,7 \%)$ patients a neuropathic component of pain was revealed.

The relationship was established between the presence of NCP and the severity of anxiety $(r=0,27, p<0,05)$, depression $(r=0,31, p<0,05)$. The relationship was revealed between the presence of NCP and the severity of pain according to the VAS $(r=0.32, p<0.05)$.

To explain the relationship between the presence of NCP and anxiety-depressive disorders, the patients were divided into two groups depending on the presence of NCP, comparable in age, the main clinical characteristics of RA, and basic therapy. The level of anxiety in women with NCP $(9,5[7 ; 13])$ was significantly higher than in patients without NCP $(6.1[4 ; 9])(p=0.01)$. The severity of depression in women with NCP was $8,55[6 ; 11]$, in patients without NCP $-5,15[3 ; 6]$ $(p=0.005)$.

Conclusion: Thus, every fifth patient with RA had clinically significance anxiety and depression, subclinical anxiety and depression were found in $26 \%$ of RA patients. Most of the patients $(81 \%)$ had moderate or severe pain, half of the patients had signs of neuropathic pain. The relationship between the severity of anxiety and depression with the intensity of pain and the presence of a neuropathic component was revealed

Disclosure of Interests: None declared

DOI: 10.1136/annrheumdis-2021-eular.2795

\section{AB0865-HPR FREQUENCY OF DEPRESSION IN SYSTEMIC LUPUS ERYTHEMATOSUS}

L. Cano Garcia ${ }^{1}$, S. Manrique Arija ${ }^{1}$, F. Godoy-Navarrete ${ }^{1}$, A. M. CabezasLucena', G. Diaz-Cordobes ${ }^{1}$, N. Mena-Vázquez ${ }^{1} .{ }^{1}$ Hospital Regional Universitario de Málaga, Rheumatology, Málaga, Spain

Objectives: Cross-sectional observational study of a series of SLE patients selected from the Rheumatology consultations.

Methods: age $\geq 18$ years with SLE (ACR 1997 criteria) capable of understanding and willing to take the questionnaires. Protocol: All patients with SLE undergoing follow-up in the rheumatology clinic are recorded in a database. A telephone call was made to all the patients included in the database and those patients who responded to the call and gave their verbal consent for the collection of data from their clinical history and completed the Goldberg questionnaire were finally included. The nurse was in charge of explaining the questionnaire to the patients. Variables: the main outcome variable was depression assessed by Goldberg ( $\geq 2$ depression) and other variables were: previous diagnosis of depression, Charlson index, polypharmacy, psychiatric medication, referral to mental health or primary care, SLEDAI and SLICC. Descriptive, bivariate statistical analysis and multivariate logistic regression analysis (VD: Goldberg depression).

Results: 89 patients with SLE were included (95.5\% women, mean age $49.44 \pm$ 13.2 years and $18.28 \pm 9.19$ years of disease). The mean (SD) of the Goldberg scale in all the patients was $3.2 \pm 2.9$ and a total of 45 patients $(50.4 \%)$ met criteria of depression according to Goldberg's screening, of which 19 (21.3\%) patients had a previous diagnosis of depression. Only 9 patients $(10.1 \%)$ had had a mental health follow-up and 22 patients $(24.7 \%)$ were being followed by the family doctor. A total of 87 patients $(97.8 \%)$ presented polypharmacy: severe polypharmacy $59(66.3 \%)$ and $33(37.1 \%)$ psychiatric medication. The most used psychiatric medication was: 7 (7.8\%) bromazepam, 6 (6.7\%) citalopram, 5 (5.6\%) diazepam. Regarding comorbidities, the Charlson index was $1.82 \pm 1.21$, also highlighting that $34(27 \%)$ of the sample had Sjögren syndrome. In the multivariate analysis, polypharmacy (OR, $1.8[95 \% \mathrm{Cl}, 1.0-3.1])$ and Sjogren's syndrome (OR, 3.8 [95\% Cl, 1.0-10.7]) were independently associated with depression by Goldberg.

Conclusion: Depression is underdiagnosed and undertreated in patients with SLE. Depression is associated with polypharmacy and the perception of patients with SLE of being ill. It is important to correctly treat depression in the context of SLE comorbidity due to its great impact on quality of life.

Disclosure of Interests: None declared

DOI: 10.1136/annrheumdis-2021-eular.3204

\section{HPR Epidemiology and public health (including prevention)}

\section{AB0866-HPR INPATIENT RHEUMATOLOGY HEALTHCARE IN A TERTIARY REFERRAL ACADEMIC HOSPITAL}

C. M. Gamboa-Alonso ${ }^{1}$, G. Figueroa-Parra ${ }^{1}$, A. L. De-Leon-Ibarra ${ }^{1}$, J. DíazAngulo $^{1}$, G. Serna-Peña ${ }^{1}$, D. Vega-Morales ${ }^{1}$, D. Á. Galarza-Delgado ${ }^{1}$.

${ }^{1}$ Universidad Autónoma de Nuevo León, Reumatología e Inmunología Clínica, Monterrey, Mexico

Background: Diagnosis of autoimmune diseases (AD) has been increasing in recent years, with a prevalence of $3-5 \%$ worldwide. The causes that lead to an inpatient approach in patients with suspicion or a confirmed rheumatic disease (RD) vary widely.

$\mathrm{RD}$ are at higher risk of infectious, cardiovascular and other comorbidities due to immunosuppression, a continuous inflammatory state and an altered immune response, increasing morbidity and mortality rates.

Objectives: Describe hospitalized patients who required a rheumatology consultation due to a preexistent or a new RD.

Methods: A descriptive, observational study was performed during a three year follow up (March 2017 -February 2020) in a University Hospital in Mexico. We included patients with definite or suspicion of a RD who received a rheumatologic evaluation. Demographic and clinical characteristics, length of stay and complications such as infection, readmission, use of mechanical ventilation and mortality were registered. Descriptive statistics were used Binary regression models were applied to find association between clinica factors and infection/mortality in RD hospitalized patients, SPSS v20 was used to perform statistical analysis. $A P<0.05$ was considered statistically significant.

Results: A total of 642 hospitalized patients received a rheumatologic evaluation. Of the evaluated patients, $315(49.1 \%)$ had a history of a confirmed RD, of these, $237(75.2 \%)$ were hospitalized due to a complication associated to the underlying RD and 78 were hospitalized for other non-rheumatic nor related disorder and were omitted for the rest of the analysis; $327(50.9 \%)$ patients were evaluated for the first time, $147(45.0 \%)$ of them being diagnosed with a new RD; Systemic Lupus Erythematosus (SLE) 55 (37.4\%) was the most frequent diagnosis. Figure 1

Of the 642 evaluated patients, $384(59.81 \%)$ patients with a previous (237) or new (147) rheumatic cause of hospitalization were studied. Table $1 \mathrm{~A}$

Factors associated to infection and mortality in the 384 patients are shown in Table 1B.

Conclusion: Inpatient rheumatologic evaluation is a major concern of healthcare due to the severity and prognosis of these diseases. SLE was found to be the most common diagnosis in patients evaluated for the first time. Infection is an important cause of hospitalization and mortality, being the use of steroids and immunosuppression some of the main risk factors of these outcomes.

\section{REFERENCES:}

[1] O'Sullivan O, Bateman J, Jobanputra P. 172 Acute Rheumatology Referrals are Increasing: A Service Evaluation of more than 1000 Consecutive Acute Inpatient Referrals from a Tertiary Centre. Rheumatology. 1 de abril de 2016;55(suppl_1):i131-2. 